\title{
White Blood Cell Differentiation Using a Solid State Flow Cytometer ${ }^{1}$
}

\author{
R.M.P. Doornbos, E.J. Hennink, C.A.J. Putman, B.G. De Grooth, and J. Greve \\ Department of Applied Physics, Cell Characterization Group, University of Twente, 7500 AE Enschede, \\ The Netherlands
}

Received for publication July 31, 1992; accepted March 16, 1993

\begin{abstract}
A flow cytometer using a solid state light source and detector was designed and built. For illumination of the sample stream two types of diode lasers $(670 \mathrm{~nm}$ and $780 \mathrm{~nm}$ ) were tested in a set-up designed to differentiate human leukocytes by means of light scattering. The detector is an avalanche photodiode, which was used to detect the weak scattered light in the orthogonal direction. The new flow cytometer set-up is very small, relatively
\end{abstract}

cheap and yields similar results as a standard flow cytometer set-up using a helium-neon laser and photomultipliers. (c) 1993 Wiley-Liss, Inc.

Key terms: Instrumentation, diode laser, avalanche photodiode, miniaturization, differential white blood cell count, light scattering, flow cytometry, immunofluorescence
Up till now all commercial and home built flow cytometers use gas lasers or arc lamps as light sources. Progress in the field of optical solid state components has led to the development of the diode laser. This chipsize laser is now available at wavelengths down to 635 $\mathrm{nm}$ having an output power of about $3 \mathrm{~mW}$. Although the wavelengths and powers of the currently available diode lasers can not be used for fluorescence measurements, they should be appropriate for light scattering measurements. An important application of light scattering measurements is the white blood cell differentiation (7). This application would benefit substantially from the advantages of the diode laser, namely low costs and small size. We have investigated the use of such a diode laser as a light source in a flow cytometer for white blood cell differentiation.

The size and costs of the system can be further reduced by replacement of the photomultiplier by its solid state analog, the avalanche photodiode. Also the replacement of the microscope objective by a compact disk lens for detection of orthogonal light scattering will reduce size and costs.

In this article we describe such a system and discuss the possibilities for white blood cell differentiation and (immuno-)fluorescence measurements.

\section{MATERIALS AND METHODS}

White blood cells were obtained using the fast lysing procedure described previously (7). Red blood cells are lysed by adding $800 \mu l$ lysing reagent (Lysright Ortho
Diagnostics, USA) to $40 \mu \mathrm{l}$ of blood of healthy individuals (EDTA $\left(\mathrm{Na}_{2}\right)$, Venoject, Tokyo, Japan). After $30 \mathrm{~s}$ $800 \mu \mathrm{l}$ phosphate-buffered saline is added to the mixture to stop the lysing process and stabilize the cells.

The flow cell is the type used in the Cell Dyn 3000 hematology system (Abbott Diagnostics, Mountain View, Ca, USA). The aspherical compact disk lens (CDlens) is normally used in compact disk players and has a focal length of $4.5 \mathrm{~mm}$ and a NA of 0.4 (Philips, Eindhoven, The Netherlands).

Polystyrene microspheres $(2.02 \mu \mathrm{m})$ were obtained from Polysciences, Inc. Warrington, PA, USA.

\section{DIODE LASERS}

Two types of diode lasers were used: the Hitachi HL7838G (Hitachi, Tokyo, Japan) with a GaAlAs double heterojunction structure with $20 \mathrm{~mW}$ of optical power at $780 \mathrm{~nm}$ in a single longitudinal mode, and the Hitachi HL6711G, with a GaAlInP, gain-guided, double heterojunction structure and $5 \mathrm{~mW}$ of optical power at $670 \mathrm{~nm}$ in multiple longitudinal modes. The light output of the diodes is stabilized using the photocurrent of the internal photodiode as a feedback signal for the power supply.

\footnotetext{
${ }^{1}$ This research was supported by Abbot Diagnostics (formerly Unipath/Sequoia Turner Corporation), Mountain View, CA, USA.
} 
An important characteristic of a diode laser is that it emits a highly divergent beam. The divergence of the beam in the direction parallel to the emitting stripe of the semiconductor device is different from that in the perpendicular direction, which results in an elliptical cone. For the currently available diode lasers the aspect ratio (ratio of the long and small axis of the elliptical spot) ranges from 2.5 to 5 (depending on the type of diode laser). Typical values for the divergence angles are $30^{\circ}$ and $8^{\circ}$ (half of the total cone angle, $\mathrm{e}^{-2}$ points). This difference in beam divergence seems a disadvantage at first sight, but in a flow cytometer we can use this phenomenon to our advantage. Because of the elliptical cone a single lens is in principle sufficient to make an elliptical spot, as desired in a flow cytometer. According to Gaussian beam optics the direction in which the divergence is larger will have the smaller waist dimensions, and vice versa. In a flow cytometer the long axis of the elliptical spot must be chosen perpendicular to the sample stream, in order to get an optimal illumination profile (5), so the emitting stripe of the diode laser is oriented horizontally.

It should be noted that since a diode laser suffers from astigmatism, the simple description assuming Gaussian profiles does not hold any more. In particular the intensity distribution near the focal region can be rather complicated $(1,6)$. However, as will be shown in the Results, a useful profile can be obtained close to the focal points.

\section{AVALANCHE PHOTODIODES}

In most flow cytometers orthogonally scattered light is collected by a microscope objective and detected with a photomultiplier. With a low power laser as light source the intensity of this scattered light is too low to be detected by a normal photodiode. This is due to the noise introduced by the amplifier following the photodiode. This amplifier noise can be eliminated by using an internal amplification mechanism. In a photomultiplier the internal gain mechanism is almost free of noise, which means that the largest noise contribution is generated by either photon statistics of the signal, dark current or background. In flow cytometry the dark current noise of a photomultiplier is negligible due to the short measuring times. There also exist photodiodes with internal gain, avalanche photodiodes (APDs), but they have more noise than a photomultiplier.

When only signal and dark current noise are considered, the signal-to-noise ratio ( $\mathrm{S} / \mathrm{N}$ ratio) of both detectors can be described by $(8,9)$

$$
\mathrm{S} / \mathrm{N}=\frac{\eta \cdot \mathrm{N}_{\mathrm{s}}}{\sqrt{\mathrm{F} \cdot \eta \cdot\left(\mathrm{N}_{\mathrm{s}}+\mathrm{N}_{\mathrm{d}}\right)}}
$$

In this formula $\mathrm{N}_{\mathrm{s}}$ and $\mathrm{N}_{\mathrm{d}}$ are the number of incident signal photons and dark photons in $\tau$ seconds (in this case $\tau$ is the transition time of the cell). $F$ is the excess noise figure and $\eta$ the quantum efficiency of the detector. In Figure 1 the S/N ratios of the APD, PMT and the ideal detector are plotted against the incident number of photons (in $5 \mu \mathrm{s}$ ) for two wavelengths. The $\mathrm{S} / \mathrm{N}$ ratio of the APD is slightly higher than that of the PMT for $780 \mathrm{~nm}$ at higher intensities, whereas at $670 \mathrm{~nm}$ it is almost equal. For the very low intensities the PMT is better in both cases. This can also be seen in Table 1 , noise characteristics, where we have calculated the minimum detectable signal power for both detectors: it is clear that the photomultiplier can detect lower light levels in the visible region.

The silicon avalanche photodiode used was an RCA C30902S (RCA Inc., Vaudreuil, Canada). In Table 1 the specifications of this APD are compared to that of a photomultiplier often used in flow cytometry (Hamamatsu R928).

Because of the strong temperature dependent gain factor of an APD, we had to stabilize the temperature $\left(35^{\circ} \mathrm{C}\right)$ using a feedback circuit with a thermoelectric heater/cooler (Peltier element). This circuit was able to keep the change of the gain within $0.75 \%$ per degree ambient temperature change, which is good enough for measurements of normal duration.

\section{INSTRUMENT DESIGN Diode Laser and Photomultipliers}

To illuminate the flow cell it is possible to use only one lens to collimate the laser light and to focus the light onto the flow cell. The size of the illumination spot is determined by the divergence of the emitted laser light, the distance from lens to cell and the focal length of the lens. Experiments with this set-up showed that high stability demands are put on the position of the lens. A second possibility for illumination is to use one lens to collimate the divergent beam for the diode laser and one spherical lens or a combination of lenses (two cylinder lenses or a cylindrical and a spherical lens) to focus this collimated beam.

The first experimental setup that was used to test the illumination of the second type is depicted in Fig. ure 2. The light from the diode laser (Hitachi HL6711G, $670 \mathrm{~nm}$ ) is collimated by a CD-lens. In order to identify eosinophilic granulocytes by measuring depolarized orthogonal light scattering, we have to use a vertically polarized illumination (2). The laser is oriented to get an elliptical spot perpendicular to the sample stream, yielding an unwanted horizontal polarization. Vertical polarization was obtained by using a retarder. This half-wave plate is followed by a linear polarizer to eliminate all residual horizontally polarized light. Two cylindrical lenses (with focal length 90 $\mathrm{mm}$ resp. $25 \mathrm{~mm}$ ) focus the collimated beam at the flow cell to a spot size of $75 \mu \mathrm{m}$ by $5 \mu \mathrm{m}$. The power of the beam incident on the flow cell is about $2 \mathrm{~mW}$. A PIN diode (model PIN 10.D, United Detector Technology, Santa Monica, CA, USA) detects the forward scattered light from $1^{\circ}$ to $3^{\circ}$. The scattered light in the orthogonal direction is collected by a Leitz H32 microscope objective with NA $=0.6$ (Wild Leitz, Wetzlar, Germany). A diaphragm in the image plane of the sample stream is 

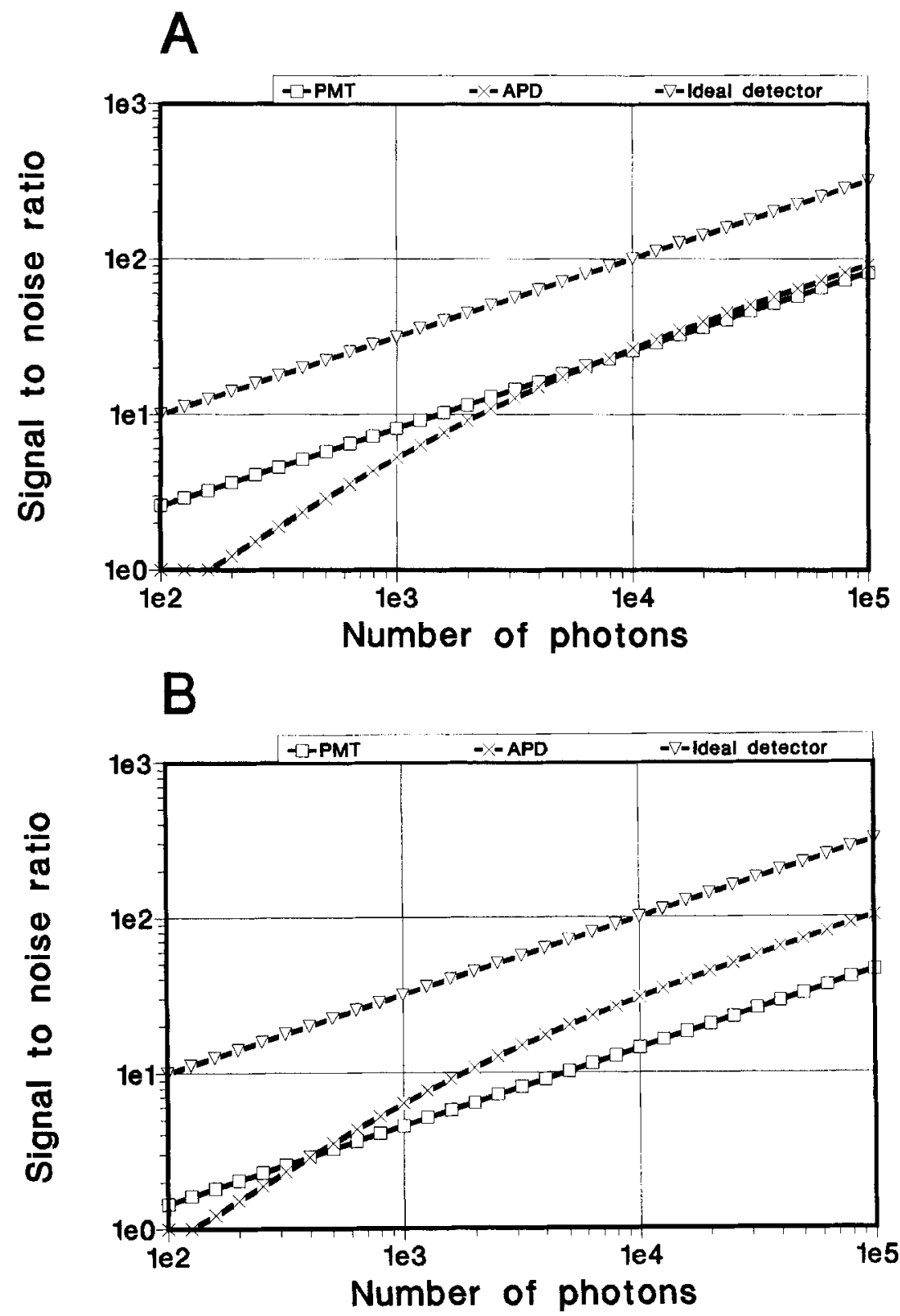

FIG. 1. The signal-to-noise ratios of the APD, PMT and an ideal detector are plotted against the incident number of photons during illumination $( \pm 5 \mu \mathrm{s})$ for a laser wavelength of $670 \mathrm{~nm}(\mathbf{A})$ and 780 $\mathrm{nm}$ (B).

used to obstruct the scattered light originating from the quartz-water interfaces. Two photomultipliers (Hamamatsu, type R928) are used to detect the total and the depolarized orthogonal light scattering in this set-up.

\section{Diode Laser and Avalanche Photodiodes}

A significant further reduction in size of the instrument described in the previous section is obtained when the photomultipliers are replaced by avalanche photodiodes (APDs). This was done with the second setup, shown in Figure 3. The light from the diode laser
(Hitachi HL7838G, $780 \mathrm{~nm}$ ), collimated by a CD-lens, is focused by a spherical lens ( $f=40 \mathrm{~mm}$ ). In order to increase the horizontal dimension of the focus we added a cylindrical lens ( $f=200 \mathrm{~mm}$ ).

In order to reduce the size (and costs) further, we replaced the Leitz objective by the small CD-lens (Philips, NA $=0.4, f=4.5 \mathrm{~mm}$ ). Because of the small sensitive area of APDs $(0.5 \mathrm{~mm}$ in diameter), CD-lenses are positioned in front of them to focus the scattered light. This "all solid state" flow cytometer occupies a space of $15 \times 15 \times 5 \mathrm{~cm}^{3}$ (see Fig. 4). 
Table 1

Characteristics of an Avalanche Photodiode and a Photomultiplier ${ }^{\mathrm{a}}$

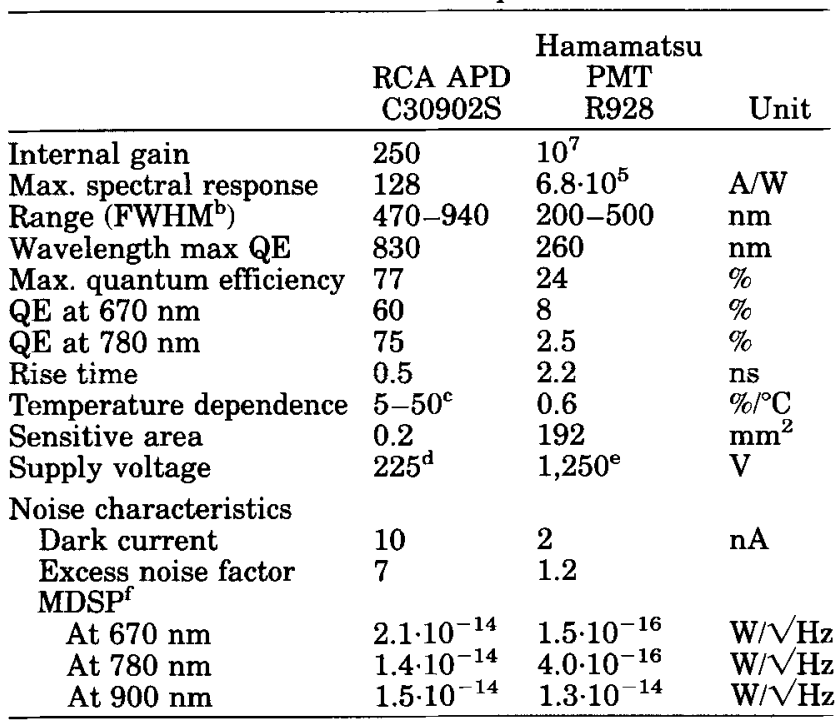

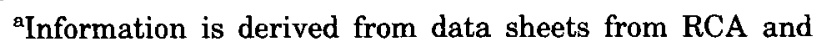
Hamamatsu.

${ }^{\mathrm{b}} 50 \%$ points of maximum $\mathrm{QE}$.

'Strongly dependent on the reverse voltage.

${ }^{\mathrm{d}}$ Breakdown voltage.

Maximum voltage.

${ }^{f}$ Minimum detectable signal power; $\mathrm{S} / \mathrm{N}=1$.

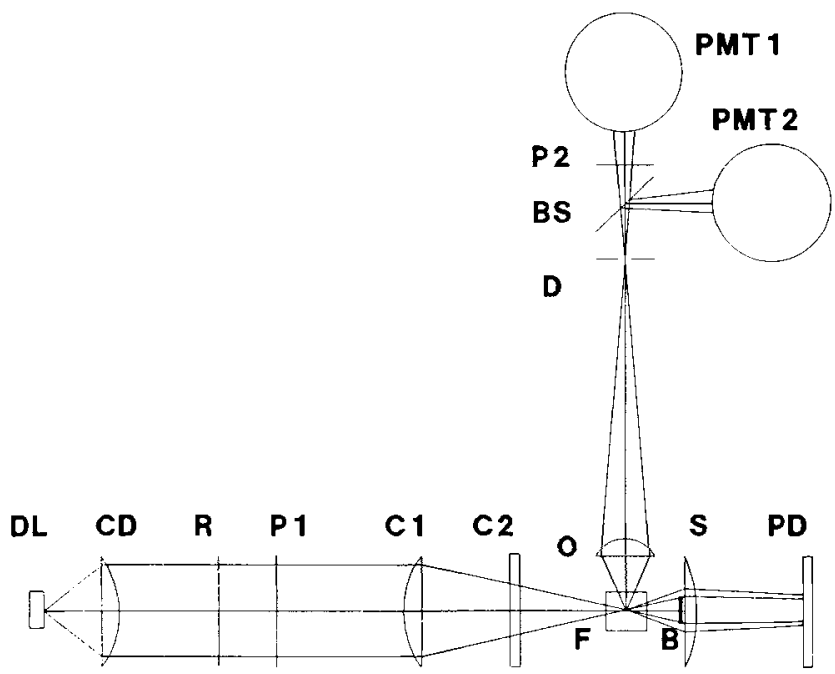

FIG. 2. Setup of a flow cytometer using a diode laser. DL, diode laser; $C D$, compact disk player lens; $R$, half-wave retarder; $P 1, P 2$, polarizers; $\mathrm{C} 1, \mathrm{C} 2$, cylindrical lenses; $\mathrm{F}$, flow cell; O, objective; B, beam stop; S, spherical lens; PD, photodiode; D, diaphragm; BS, beam splitter; PMT1,PMT2, photomultiplier (drawing not to scale).

\section{RESULTS}

Because of astigmatism of the diode laser the intensity distributions in the focal region are not Gaussian $(1,6)$. These illumination profiles occurring at positions out of focus are indeed observed, see Figure 5. How-

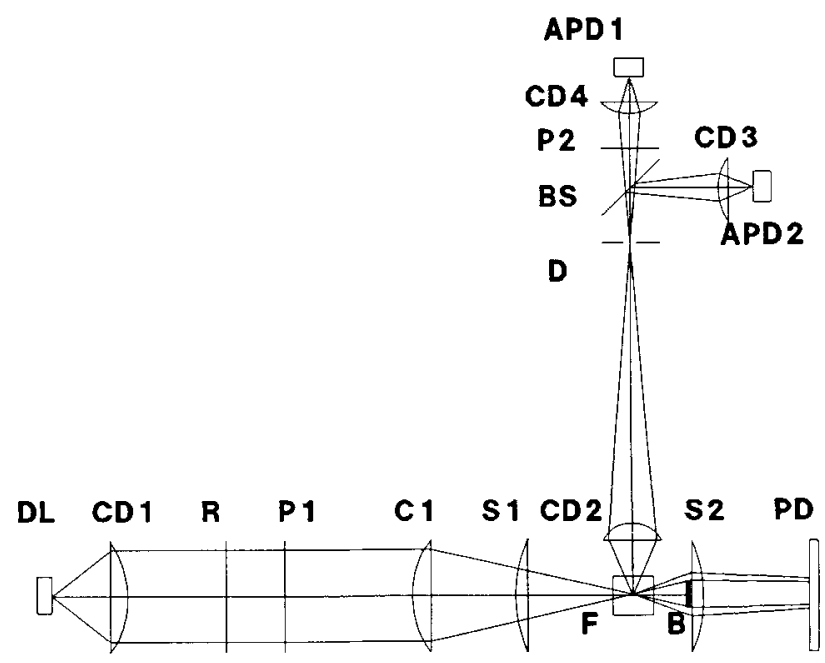

FIG. 3. Layout of a flow cytometer using a diode laser and two avalanche photodiodes for detection in the orthogonal direction. DL, diode laser; CD1,CD2,CD3,CD4, CD-lenses; R, retarder; P1,P2, polarizers; $\mathrm{C} 1$, cylindrical lense; F, flow cell; O, objective; $\mathrm{B}$, beam stop; $\mathrm{S} 1$, S2, spherical lenses; PD, photodiode; $D$, diaphragm; BS, beam splitter APD1,APD2, avalanche photodiodes (drawing not to scale).

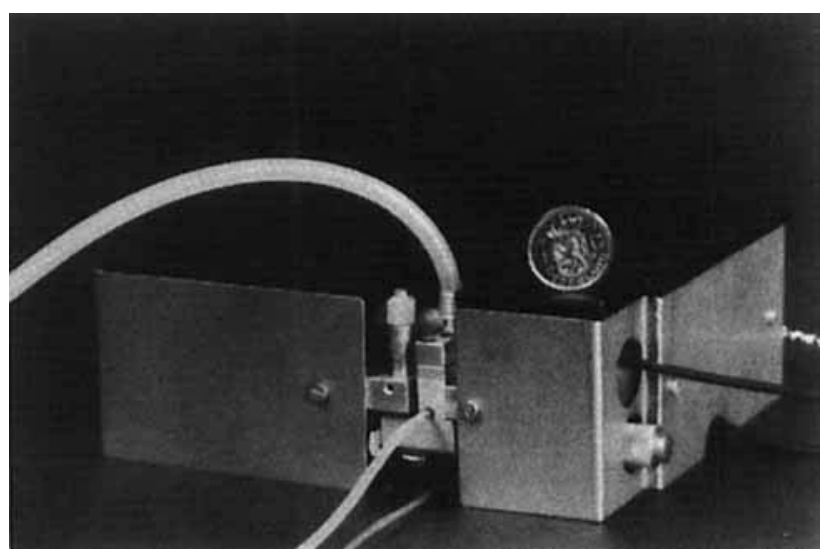

Fig. 4. Photograph of set-up using a diode laser as a light source and avalanche photodiodes for detection in the orthogonal direction. The optical path has been cut out of a block of aluminum. All mentioned parts are firmly attached to the cut out region, still diode laser, flow cell and both APDs can be adjusted with simple positioners.

ever, the profile at the focus (profile C) is approximately Gaussian and serves our purpose well. The profile in the other direction is shown in Figure 6. The flat top of the profile, due to the cylindrical lens allows measurements of large core diameters (here about 40 $\mu \mathrm{m})$.

The results of measurements of $1.0 \mu \mathrm{m}$ microspheres are fairly good: we obtained with the second set-up a $\mathrm{CV}$ of $2.2 \%$ in the side scattering signals. When we used a $1 \%$ transmission filter the noise was determined by signal noise and the CV increased to $7.3 \%$. The first set-up gave similar results. The forward light scatter- 


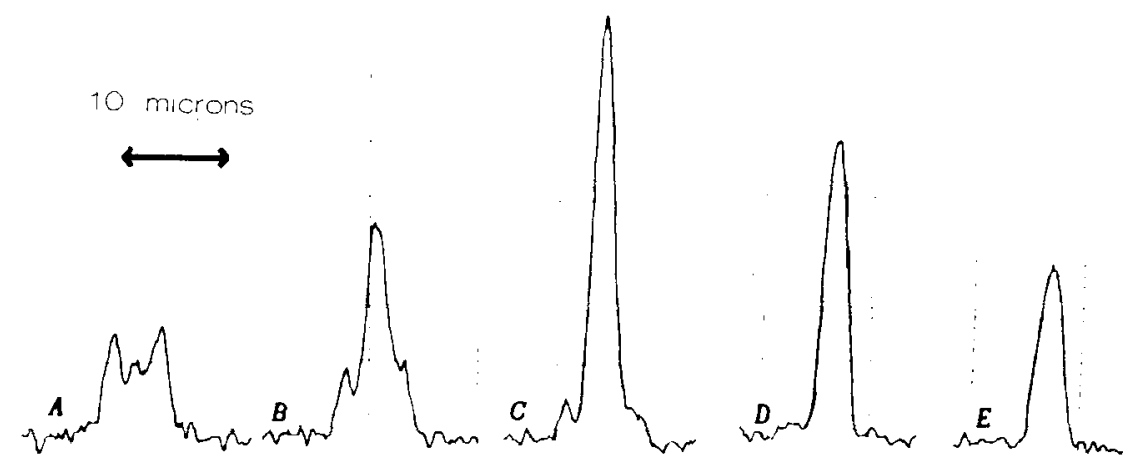

FIG. 5. Near focus intensity profiles in the direction of the flow. The curves were obtained by measuring the forward light scattering of small polystyrene beads $(2.02 \mu \mathrm{m})$ as a function of time. Profiles at different positions of the laser beam were obtained by placing the

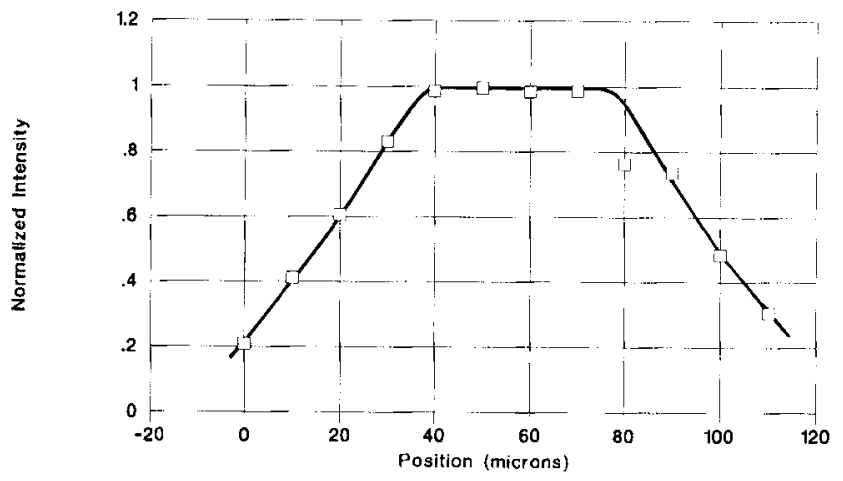

Fig. 6. Illumination profile in the direction perpendicular to the laser beam and sample stream (this is the long axis of the elliptical spot). The curves were obtained by measuring the forward light scattering of small polystyrene beads $(2.02 \mu \mathrm{m})$ as a function of the position. Mark the flat top of the profile.
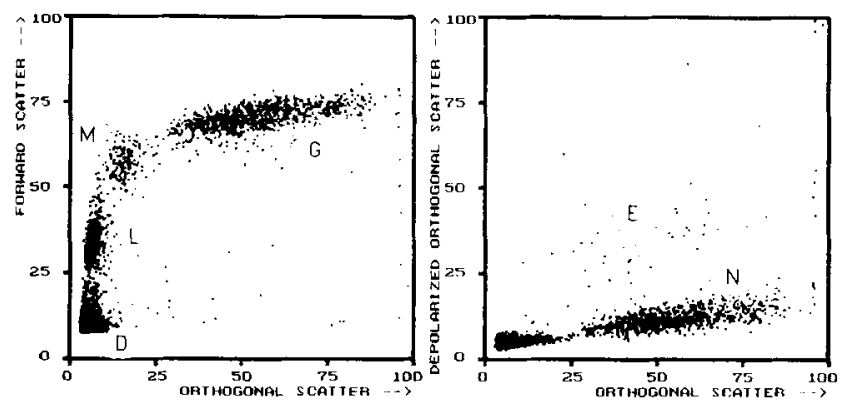

Fig. 7. Scatter plots of human white blood cells obtained with a flow cytometer using a diode laser as a light source and PMTs for side scatter detection. L, lymphocytes, M, monocytes, G, granulocytes, N, neutrophils, E, eosinophils, D, debris.

ing of $1.0 \mu \mathrm{m}$ microspheres yields a CV of $11.0 \%$ which is determined by the large background originating from scattering of impurities in the cuvette.

In Figure 7 the results of a blood cell differentiation are shown which were obtained using the first setup. particle stream in the flow cell at the indicated positions. Profiles $\mathbf{A}$ and $\mathbf{B}$ are 4 and $2 \mathrm{~mm}$, respectively, in front of the focus, profile $\mathbf{C}$ is exactly in focus and profiles $D$ and $E$ are 2 and $4 \mathrm{~mm}$ behind the focus.

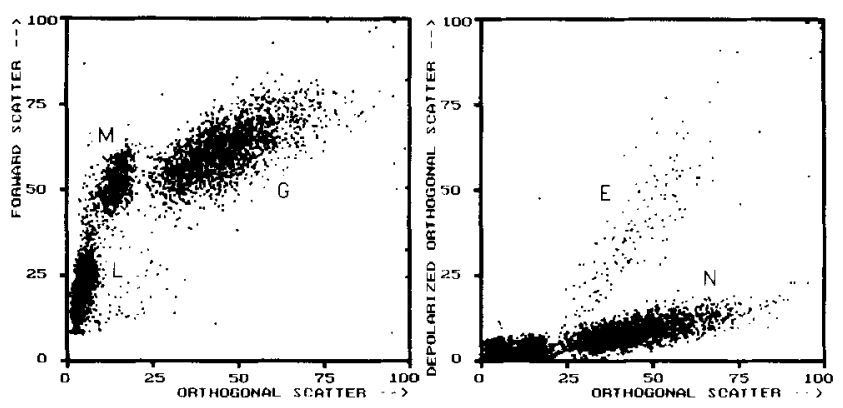

FIG. 8. Scatter plots of human white blood cells obtained with a flow cytometer using a diode laser as a light source and avalanche photodiodes for detection in the orthogonal direction. L, lymphocytes, M, monocytes, G, granulocytes, N, neutrophils, E, eosinophils.

The scatter plots show four populations which we tentatively ascribe to the lymphocytes, monocytes, neutrofilic and eosinophilic granulocytes. Comparison with a $\mathrm{He}-\mathrm{Ne}$ equipped system described previously (7) confirmed this interpretation. Thus a 4-part differential white blood cell count is possible using a diode laser for illumination.

Similar measurements on human blood obtained with the second set-up are shown in Figure 8. From the scatter plots it is clear that a 4-part differential white blood cell count is possible using a diode laser for illumination and APDs for low intensity orthogonal scattering detection. The definition of the different populations is as good as in a setup using a He-Ne laser and PMTs (7). Even the very low intensity depolarized scattering channel gives a sufficient signal-to-noise ratio, as can be deduced from the good separation between the neutrophilic and eosinophilic granulocytes.

An important aspect of automatic differentiation of white blood cells is the number of cells that be analyzed per second, the throughput. The throughput is limited by the relatively low concentration of the sample, due to the necessary dilution caused by the lysis procedure. Given an optimal lysis procedure (minimal dilution) 
and maximum flow velocity (about $10 \mathrm{~m} / \mathrm{s}$ ), a high throughput can only be achieved with a relatively large flow core diameter. With this system a maximum throughput of about 1,000 cells $\mathbf{s}^{-1}$ was obtained, allowing $5 \%$ variation in laser intensity.

\section{DISCUSSION}

The experiments show that differentiation of the human white blood cells can be done with diode lasers and APDs resulting in good discrimination of the cell populations. The resolution expressed in the quality of the separation of the different populations is comparable to set-ups using He-Ne lasers or argon ion lasers and photomultipliers.

Both the $670 \mathrm{~nm}$ laser and the $780 \mathrm{~nm}$ laser give good scatter plots, although the positions of the cell populations are shifted. This is the result of the use of different forward and side scattering detectors in the experiments and of the change in wavelength $(3,4)$. When we compared our experiences with the $780 \mathrm{~nm}$ and $670 \mathrm{~nm}$ lasers, we prefer the $670 \mathrm{~nm}$ laser, because the light is clearly visible which facilitates the alignment considerably. Although the $780 \mathrm{~nm}$ laser had 20 $\mathrm{mW}$ and the $670 \mathrm{~nm}$ laser had only $5 \mathrm{~mW}$ of optical power, the observed signal-to-noise ratio was more than sufficient.

The APDs performed very well in our systems, but they have two major disadvantages: the large temperature dependence and the small size of the sensitive area, which requires precise focusing of the scattered light. We solved the first problem by stabilizing the temperature externally. In future this problem will be solved when APDs with built-in temperature regulation will become commercially available. The second problem will be eliminated by using APDs with large sensitive areas (up to $175 \mathrm{~mm}^{2}$, recently introduced by Advanced Photonix Inc. Camarillo, CA, USA), although they probably would have a higher dark current noise. As can be seen from Figure 1, the APD can not be used to replace the PMT for detection of weak fluorescence measurements in the visible region.

In the second set-up we used a CD-lens to collect scattered light in the orthogonal direction. The CD lens is a very cheap high-quality aspherical lens, originally designed for collimation and focusing $780 \mathrm{~nm}$ light from diode lasers for compact disk readout. When used to collect scattered light its numerical aperture of 0.4 is enough, but for future fluorescence measurements a higher NA value would be required.

There are several advantages with a flow cytometer containing APDs, CD-lenses, and a diode laser. First, the flow cytometer system can be very small (see Fig. 4). Second, the components used are very cheap. No high-voltage power supplies are needed for the APDs: a voltage of $200 \mathrm{~V}$ suffices.

The value of the system would increase enormously if immunofluorescence measurements would become possible using a diode laser system. The problem of using diode lasers for excitation of fluorescence is that available diode lasers have wavelengths which are unsuitable for the currently available dyes. In principle, allophycocyanine (APC), can be excited with the 635 nm diode laser (Power Technology Inc., Mablevale, AR, USA), but the power is still too low ( $3 \mathrm{~mW}$ ). A suitable dye for the described system is Ultralite 720 (Ultralite Corporation, USA; not available anymore), which can be excited at $670 \mathrm{~nm}$ and has its emission around 720 $\mathrm{nm}$.

Our first attempts to measure biotin-CD3 labeled lymphocytes with streptavidin-Ultralite 720 gave disappointing results. Although Ultralite 720 fluorescence was measured, the high non-specific staining prevented distinction between CD3-positive and CD3negative lymphocytes (data not shown). With a large effort in carefully optimizing the measurements it may be possible to get a separation, but better dyes or more powerful diode lasers with low wavelengths are needed (recently $100 \mathrm{~mW} 670 \mathrm{~nm}$ lasers were presented at SPIE ' 92 by Power Technology Inc., Mablevale, AR, USA.).

\section{CONCLUSIONS}

We have shown that it is possible to design and operate a flow cytometer using a diode laser for illumination and avalanche photodiodes for the detection of low intensity scattered light. The results are comparable to the data obtained from a conventional flow cytometer. The major advantages of this new system are its small size and low costs. Immunofluorescence detection may be possible in the future if better red-excitable dyes and diode lasers with higher powers at lower wavelengths become available.

\section{LITERATURE CITED}

1. Asbeck PM, Cammack DA, Daniele JJ: Non-Gaussian fundamental mode patterns in a narrow-stripe-geometry lasers. Appl Phys Lett 33(6):504, 1978.

2. De Grooth BG, Terstappen LWMM, Puppels GJ, Greve J: Lightscattering measurements as a new parameter in flow cytometry. Cytometry 8:539, 1987.

3. De Grooth BG, Van Dam M, Swart NC, Willemsen A, Greve J: Multiple wavelength illumination in flow cytometry using a single arc lamp and a dispersing element. Cytometry 8:445, 1987.

4. Loken MR, Houck: Light scattered at two wavelengths can discriminate viable lymphoid cell populations on a fluorescence activated cell sorter. J Histochem Cytochem 29:609, 1981

5. Shapiro HM: Practical Flow Cytometry, Ed. 2. Alan R. Liss, Inc., New York, 1988.

6. Tatsuno K, Arimoto A: Measurement and analysis of diode laser wave fronts. Appl Optics 20(20):3520, 1981.

7. Terstappen LLWM, De Grooth BG, Visscher K, Van Kouterik FA, Greve J: Four-parameter white blood cell differential counting based on light scattering measurements. Cytometry 9:39, 1988.

8. Webb PP, McIntyre RJ, Conradi J: Properties of avalanche photodiodes. RCA Review 35:234, 1974.

9. Wilson J, Hawkes JFB: Optoelectronics, Ed. 2. Prentice Hall, New York, 1989 